\title{
Borderline and Malignant Phyllodes Tumors: How Often do They Locally Recur and is There Anything we can do About it?
}

\author{
Richard J. Barth Jr., MD \\ Department of Surgery, Dartmouth Hitchcock Medical Center, Lebanon, NH
}

For the past 10 years, I have received an automatic monthly email from my librarian with new papers published on phyllodes tumors (PTs). Although these are unusual neoplasms, investigators like to study them; I commonly have a couple of abstracts to read each month. Except for one prospective study, the literature on PTs consists of retrospective studies of clinical outcomes or clinical-pathologic associations. Just about everyone agrees about certain aspects of surgical treatment: (1) the local recurrence (LR) rate is much lower when negative margins are achieved; (2) borderline and malignant PTs locally recur with equal frequencies; and (3) distant metastases occur in 10-20\% of patients after resection of malignant PTs, and rarely after borderline phyllodes resections. In the current issue of Annals of Surgical Oncology, Dr. Spanheimer and associates elegantly describe the clinical outcomes of a large cohort of borderline and malignant phyllodes patients (124 patients over a 60-year period) and provide data that confirms concepts $1-3$. $^{1}$

A critical question in the management of patients with borderline and malignant PTs is what is the LR rate after margin-negative (no tumor on ink) breast-conserving surgery (BCS)? It is difficult to gather this data because few authors provide enough detail in their papers to determine the LR rate in patients with borderline or malignant PTs who underwent BCS with negative margins. In 2009, the results of 13 studies were summarized: 12/50 patients (24\%) with borderline PTs and 25/124 patients (20\%) with malignant PTs locally recurred after margin-negative BCS. $^{2}$ Since then, five additional studies have reported

(C) Society of Surgical Oncology 2019

First Received: 12 January 2019;

Published Online: 12 March 2019

R. J. Barth Jr., MD

e-mail: Richard.J.Barth.Jr@Hitchcock.ORG their findings with sufficient detail to answer this question. Rodrigues et al. found four LRs in 15 patients (27\%); Kim et al. identified LRs in 9/37 patients (24\%); Choi et al. identified LRs in 37/230 patients (16\%); Zhou et al. identified LRs in 45/197 patients (23\%); and Onkendi et al. found $8 \mathrm{LRs}$ in 32 patients $(25 \%) .^{3-7}$ Combining these results with the 13 previous studies identified 140 LRs in 685 patients, a $20.4 \%$ LR rate in patients with borderline or malignant PTs undergoing BCS with negative margins.

Most would agree that an LR rate of $20 \%$ is too high and warrants consideration of adjuvant therapy. Several studies have demonstrated that adjuvant radiation therapy decreases LR rates. Two large, retrospective studies showed a statistically significant decrease in LR rates with adjuvant radiation therapy. ${ }^{8,9}$ In a report from the Rare Cancer Network of 159 patients with borderline or malignant PTs, 109 were treated with BCS and 50 underwent mastectomy; ${ }^{8} 36$ received adjuvant radiation therapy. The percentage of patients free of LR at 10 years, in the group that received radiation therapy, was significantly better than the group that did not receive radiation therapy $(86 \%$ vs. $59 \%, p=0.02$ ). Using data from the National Cancer Database, Gnerlich et al. ${ }^{9}$ showed that adjuvant radiation therapy decreased the risk of LR after resection of PTs by more than half (hazard ratio [HR] $0.43,95 \%$ confidence interval [CI] 0.19-0.95). A meta-analysis of the effect of adjuvant radiation therapy on borderline and malignant PTs, citing six non-randomized studies of 2058 patients, demonstrated a decreased risk of LR in patients with borderline and malignant PTs who received adjuvant radiation therapy after BCS (HR 0.31, 95\% CI - 0.10-0.72). ${ }^{10}$ In a prospective, multi-institutional study, 46 patients (30 with malignant PTs and 16 with borderline PTs) underwent margin-negative resections followed by radiation therapy. ${ }^{2}$ Eight of these patients had margins $<2 \mathrm{~mm}$. After 10 years of observation for all patients, none had developed an LR. Thus, there are substantial data supporting a 
beneficial effect of adjuvant radiation therapy on LR of borderline and malignant PTs. Given the potential for malignant PTs to metastasize, and the lack of effective systemic treatment for metastatic malignant PT, LRs should not be taken lightly. Surgeons and radiation oncologists should discuss with patients the risks and benefits of adjuvant radiation therapy after BCS of borderline and malignant PTs.

Another critical surgical question is how wide should the margin be when resecting borderline or malignant PTs? The current National Comprehensive Cancer Network (NCCN) guidelines recommend excision with the intention of obtaining surgical margins $\geq 1 \mathrm{~cm} .{ }^{11}$ Two papers are commonly quoted as providing supportive data for obtaining at least a $1 \mathrm{~cm}$ negative margin, but neither are conclusive because both studies include patients with positive margins in the $<1 \mathrm{~cm}$ comparison group. Kapiris et al. ${ }^{12}$ reported an LR rate of $60 \%$ in 10 patients who had positive or $<1 \mathrm{~cm}$ margins, compared with an LR rate of $28 \%$ in 14 patients whose margins were $\geq 1 \mathrm{~cm}$. Asoglu et al. ${ }^{13}$ combined 22 patients with BCS and 28 with mastectomy; nine patients had positive or $<1 \mathrm{~cm}$ margins, while the remainder had $>1 \mathrm{~cm}$ margins. The overall LR rate was $32 \%$; it was reported to be significantly less in the group with margins $>1 \mathrm{~cm}$, but even in this group the overall LR rate was $25 \%$. Thus, there are little conclusive data demonstrating that $\mathrm{a} \geq 1 \mathrm{~cm}$ negative margin leads to a lower LR than $\mathrm{a}<1 \mathrm{~cm}$ negative margin. Furthermore, the LR rate in patients in these studies who had margins $>1 \mathrm{~cm}$ is still quite high (25\%). Another problem with requiring a $1 \mathrm{~cm}$ margin, since the median diameter of PTs is quite large $(4-5 \mathrm{~cm})$, is that attempts to resect enough normal tissue around the tumor to achieve a minimum margin $>1 \mathrm{~cm}$ will leave many women with a poor cosmetic result.

The study by Spanheimer et al. ${ }^{1}$ in this issue describes outcomes after resections of borderline or malignant PTs with margins $>1 \mathrm{~mm}$. Overall, they identified LRs in $16 \%$ of 71 patients with borderline or malignant PTs undergoing BCS. Some of these patients had a positive or close $(<1 \mathrm{~mm})$ margin. Although they did not report the LR rate after no-tumor-on-ink resections, when the subset of patients with a margin $>1 \mathrm{~mm}$ was considered, the LR rate was $12 \%$. Since this LR rate is relatively low, if future studies that carefully document margin width confirm these findings, one might consider resection with a $>1 \mathrm{~mm}$ margin to be adequate therapy.

The grading of PTs as benign, borderline, or malignant is based on the World Health Organization classification. ${ }^{14}$ The histologic characteristics of number of mitoses $(<4$ vs. $>10 / 10$ high-power fields), pushing versus infiltrative borders, mild versus marked stromal atypia and cellularity, and absence versus presence of stromal overgrowth differentiate benign PTs from malignant PTs. The application of this system can be ambiguous, especially when classifying PTs as borderline versus malignant. As is evident in Table 1 of the study by Spanheimer et al., in the past some pathologists may have classified a tumor with two or three malignant characteristics as malignant, while others may have considered these tumors to be borderline. ${ }^{1}$ Spanheimer et al. make the important observation that only patients whose tumors exhibited all of the malignant characteristics (29\% of patients whose tumors were classified as malignant in their study) developed distant metastases. The disease-specific survival of this subset of patients was only $63 \%$, while patients whose tumors had less than all malignant characteristics had a disease-specific survival of $100 \%$. The suggestion by Spanheimer et al. that pathologists only classify PTs as malignant if they exhibit all the malignant features, and as borderline when only some characteristics are present, was also recommended by an expert panel of pathologists in $2016 .{ }^{15}$ In fact, the 2012 WHO definition of the grading system clearly states that "borderline PT is diagnosed when the tumor does not possess all the adverse histological characteristics found in malignant PTs". ${ }^{14}$ Understanding this distinction can help surgeons accurately discuss the likelihood of metastatic progression with our patients. Unfortunately, defining PTs as malignant only if they exhibit all malignant characteristics does not identify a subgroup at increased risk of LR. In their large study, Choi et al. ${ }^{5}$ specifically defined malignant PTs as tumors that met all of the WHO criteria, and the LR rate after BCS was similar for malignant versus borderline PTs.

While retrospective studies are important and hypothesis-generating, it is likely that future progress in the understanding of borderline and malignant PTs will come through more prospective studies of this uncommon tumor. More work also needs to be done on the value of molecular markers to predict recurrence and potentially target systemic therapies.

\section{REFERENCES}

1. Spanheimer P, Murray M, Zabor E, Stempel M, Morrow M, Van Zee $\mathrm{K}$, et al. Long term outcomes after surgical treatment of malignant/borderline phyllodes tumors of the breast. Ann Surg Oncol Epub. 2009. https://doi.org/10.1245/s10434-019-07210-4.

2. Barth R, Wells W, Mitchell S, Cole B. A prospective, multiinstitutional study of adjuvant radiation therapy after resection of malignant phyllodes tumors. Ann Surg Oncol. 2009;16:2288-94.

3. Rodrigues M, Truong P, Mcevitt E, Weir L, Knowling M, Wai E. Phyllodes tumors of the breast: the British Columbia Cancer Agency experience. Cancer Radiothe. 2018;22:112-119.

4. Kim S, Kim J, Kim D, Jung W, Koo J. Analysis of phyllodes tumor recurrence according to the histologic grade. Breast Cancer Res Treat. 2013;141:353-63. 
5. Choi N, Kim K, Shin K, Kim Y, Moon H, Park W, et al. Malignant and borderline phyllodes tumors of the breast: a multicenter study of 362 patients. Breast Cancer Res Treat. 2018;171:335-44.

6. Zhou Z, Wang C, Sun X, Yang Z, Chen X, Shao Z, et al. Prognostic factors in breast phyllodes tumors: a nomogram based on a retrospective cohort study of 404 patients. Cancer Med. 2018;7:1030-42.

7. Onkendi E, Jimenez R, Spears G, Harmsen W, Ballman K, Hieken T. Surgical treatment of borderline and malignant phyllodes tumors: the effect of the extent of resection and tumor characteristics on patient outcome. Ann Surg Oncol. 2014;21:3304-9.

8. Belkacemi Y, Bousquet G, Marsiglia H, Ray-Coquard I, Magne $\mathrm{N}$, Malard Y, et al. Phyllodes tumors of the breast. Int J Radiat Oncol Biol Phys. 2008;70:492-500.

9. Gnerlich J, Williams R, Yao K, Jaskowiak N, Kulkarni S. Utilization of radiotherapy for malignant phyllodes tumors: analysis of the National Cancer Database 1998-2009. Ann Surg Oncol. 2014;21:1222-30.

10. Zeng S, Zhang X, Yang D, Wang X, Ren G. Effects of adjuvant radiotherapy on borderline and malignant phyllodes tumors: a systematic review and meta-analysis. Mol Clin Oncol. 2015;3:663-71.
11. National Comprehensive Cancer Network. Phyllodes tumor. Version 3.2018. http://www.nccn.org. Accessed 6 Jan 2019.

12. Kapiris I, Nasir N, A'hern R, Healy V, Gui G. Outcome and predictive factors of local recurrence and distant metastases following primary surgical treatment of high-grade malignant phyllodes tumors of the breast. Eur J Surg Oncol. 2001;27:723-30.

13. Asoglu O, Ugurlu M, Blanchard K, Grant C, Reynolds C, Cha S, et al. Risk factors for recurrence and death after primary surgical treatment of malignant phyllodes tumors. Ann Surg Oncol. 2004;11:1011-17.

14. Lakhani S, Ellis I, Schnitt S, Tan P, van de Vijver M, eds. World health organization classification of tumours of the breast. Lyon: IARC Press; 2012.

15. Tan B, Acs G, Apple S, Badve S, Bleiweiss I, Brogi E, et al. Phyllodes tumors of the breast: a consensus review. Histopathology. 2016;68:5-21.

Publisher's Note Springer Nature remains neutral with regard to jurisdictional claims in published maps and institutional affiliations. 